\title{
3 Research Square

\section{Evaluation of the Therapeutic Effect of Pulmonary Embolism by CT Pulmonary Angiography Combined With Clinical Indexes}

\section{Meng Yuan}

Second Affiliated Hospital of Harbin Medical University

XinYuan Cui

Second Affiliated Hospital of Harbin Medical University

ChunXue Yang

Second Affiliated Hospital of Harbin Medical University

XinYang Zhang

Second Affiliated Hospital of Harbin Medical University

Deli Zhao

Second Affiliated Hospital of Harbin Medical University

JinLing Zhang ( $\square$ jinlingzi@163.com )

Second Affiliated Hospital of Harbin Medical University

\section{Research Article}

Keywords: Pulmonary embolism, Right ventricular function, Pulmonary angiography, Thrombolysis

Posted Date: August 3rd, 2021

DOI: https://doi.org/10.21203/rs.3.rs-672397/v2

License: () (1) This work is licensed under a Creative Commons Attribution 4.0 International License. Read Full License 


\section{Abstract}

Background To evaluate the value of CT pulmonary angiography (CTPA) in evaluating the therapeutic efficacy of pulmonary embolism (PE) through the study of CT pulmonary artery obstruction index (PAOI),right ventricular function parameters and some clinical indexes related to coagulation function and cardiac function injury.

Materials and methods Select 30 patients with pulmonary embolism who underwent CTPA examination before and after treatment in our hospital, sort out their CTPA images before and after treatment, and obtain PAOI and right heart function parameters, including ascending aorta diameter (AAd), main pulmonary artery diameter (MPAd), ratio of main pulmonary artery diameter to ascending aorta diameter (MPAd/AAd), right pulmonary artery trunk diameter (RPAd), left pulmonary artery trunk diameter (LPAd), the ratio of the maximum short axis diameter of the right ventricle to the maximum short axis diameter of the left ventricle $(\mathrm{RVd} / \mathrm{LVd})$, and calculate the pulmonary artery obstruction index (PAOI).At the same time obtain the required clinical indicators, including serum D-dimer, N-terminal B-type natriuretic peptide precursor (NT-proBNP), cardiac troponin I (cTnl), myocardial enzyme profile (aspartate aminotransferase (AST), lactate dehydrogenase (LDH), creatine kinase (CK)).

Results MPAd, RPAd, LPAd, RVd / LVd, PAOI, D-dimer, cTnl, CK, LDH, AST had significant changes after treatment $(P<0.05)$, while the other parameters and indexes had no significant differences before and after treatment $(P>0.05)$. PAOI is positively correlated with $R V d / L V d, M P A d, D$-dimer. The correlation coefficient ranges from 0.281 to 0.423 , among which, the correlation with D-dimer is the closest, with a correlation coefficient of 0.423 . There was no significant correlation between other parameters and PAOI $(P<0.05)$.

Conclusion CTPA is of great significance in evaluating the severity of pulmonary embolism, right heart function and therapeutic efficacy.

\section{Introduction}

PE is a serious venous thromboembolic disease (VTE), which can cause fatal complications. It is an important cause of death of cardiovascular related diseases. The mortality of PE patients in the first month is about $10-30 \%$. In the past decades, the use of thrombolysis, anticoagulation therapy and surgical intervention has increased, which has led to a significant improvement in the prognosis of PE patients[1-3]. In recent years, the annual incidence rate of PE and the mortality rate related to $P E$ have increased exponentially with age. Therefore, with the aging of the world population, the burden of PE on the society continues to increase[4]. With the continuous development of imaging technology, CTPA can not only intuitively detect the emboli in the arterial lumen, but also qualitatively and quantitatively diagnose the disease. This study used CTPA to obtain the right ventricular function parameters before and after treatment, and compared them with relevant clinical indicators, aiming to better evaluate the 
effect of pulmonary embolism treatment in imaging, so as to provide more basis for clinical evaluation and prognosis of pulmonary embolism.

\section{Materials And Methods}

\section{General information}

In total we identified 30 patients in our hospital who underwent CTPA examination and diagnosed PE in our hospital from January 2015 to December 2020, and received anticoagulant or thrombolytic therapy, and then perform the second CTPA examination 1-3 months after treatment.At the same time collecting some clinical indexes related to coagulation function and cardiac function injury within $24 \mathrm{~h}$ before and after CTPA examination. Among them, there were 18 males and 12 females, aged 13-83 years old, with an average of (52.67 \pm 16.67$)$. There has been no serious heart and pulmonary vascular disease and liver and kidney damage. The study was approved by the Institutional Review Board of the hospital, and informed consent was obtained from each patient.

\section{Equipment}

CTPA examination equipment: Philips brilliance ICT 256 slice spiral CT and EBW workstation Evaluate the patient's physical condition before the examination, perform routine iodine allergy tests, and introduce the purpose, methods and precautions of the CTPA examination to the patient or their family members, and sign an informed consent form. Instruct the patient to carry out breath-holding training. Each breathholding time is more than $10 \mathrm{~s}$. The patient is placed in a supine position, holding the head with both hands, and the scanning direction is from foot to head.

Scanning range: chest entrance to diaphragm level.

The scanning parameters are as follows: tube voltage $120 \mathrm{kv}$, current $250 \mathrm{mAs}, 0.5 \mathrm{~s} / \mathrm{turn}$, pitch 0.625 , inject iopromide (Uvixine $(350 \mathrm{mgl} / \mathrm{ml})$ ) through the anterior elbow vein with a double-barreled highpressure syringe, the injection rate is $5 \mathrm{ml} / \mathrm{s}$, the total dose is $60-80 \mathrm{ml}$, after the contrast agent is injected, the same flow rate is injected with the same flow rate of $0.9 \%$ sodium chloride $30 \mathrm{ml}$. The delay time is based on the artificial intelligence trigger scan, the position is set in the main lung, and the pulse trigger threshold is set to $100 \mathrm{HU}$. After scanning, the reconstructed image has a layer thickness of $0.625 \mathrm{~mm}$ and a layer spacing of $0.625 \mathrm{~mm}$. The data are transmitted to the post EBW processing workstation for analysis.

\section{Statistics indicators}

Using Qanadli embolism index[5], the calculation method is as follows, according to the two lungs are divided into 10 pulmonary segment arteries, a partial pulmonary artery blockage is counted as 1 , the complete blockage is counted as 2, and when no emboli is found, it is counted as 0 . Embolism in subsegment pulmonary artery is calculated based on partial blockage of corresponding pulmonary artery, The embolism in the artery above the pulmonary artery is equal to the total number of its branch 
pulmonary artery. For example, partial embolism of upper lobe pulmonary artery was 3 points, complete embolism was 6 points, right middle lobe and left lingual lobe pulmonary artery embolism was 2 or 4 points, bilateral lower lobe pulmonary artery embolism was 5 or 10 points, bilateral main pulmonary artery embolism was 10 or 20 points, main pulmonary artery embolism was 20 points, maximum occlusion score was 40 points. Therefore, Qanadli embolism index $=\sum(\mathrm{n} \times \mathrm{d}) / 40 \times 100 \%$, where $n$ represents the location of the obstruction with emboli, and $d$ represents the degree of obstruction score.

Measure the maximum distance from the ventricular septum to the inner side of the free wall of the ventricle on the patient's CTPA axial image, that is, the diameter of the largest short axis of the ventricle, and calculate the ratio of the maximum short axis diameter of the right ventricle to the maximum short axis diameter of the left ventricle (RVd/ LVd), simultaneously measure the diameter of the ascending aorta (AAd), the diameter of the main pulmonary artery (MPAd), the diameter of the right pulmonary artery (RPAd), and the diameter of the left pulmonary artery (LPAd), and calculate the diameter of the ascending aorta (AAd) and the diameter of the main pulmonary artery (MPAd) ) Ratio (AAd/MPAd).

Collect the clinical indicators required within 24 hours before and after the CTPA examination of the patient, including serum D-dimer, N-terminal B-type natriuretic peptide precursor (NT-proBNP), cardiac troponin I (cTnI), myocardial enzyme profile (aspartate aminotransferase (AST), lactate dehydrogenase $(\mathrm{LDH})$, creatine kinase (CK).

\section{Statistical analysis of data}

CT PAOI and CTPA right heart function parameters were completed by two senior diagnostic imaging physicians who read the CTPA images of the patient without knowing the clinical data of each patient, independently and blindly. The final results are subject to the consensus of the two physicians. Statistical analysis uses SPSS23.0 statistical software to analyze the data. The measurement data is expressed as $X \pm s$ and $[M(P 25, P 75)]$. The $K-S$ test is used to determine whether the measurement data conforms to the normal distribution. the two paired sample t test is used for those conforming to the normal distribution, and the nonparametric Wilcoxon signed rank sum test is used for those not conforming to the normal distribution, with $\mathrm{P}<0.05$ as the difference is statistically significant. Spearman rank correlation coefficient was used to evaluate the correlation between CT PAOI and RVd / LVd, AAd, MPAd, D-dimer, cTnl, NT-proBNP, AST, LDH, CK.

\section{Results}

\section{comparison of pulmonary embolism before and after treatment}

The number and degree of obstructive pulmonary segments in 30 patients before and after treatment are shown in Table 1, After treatment, CTPA showed that the area of pulmonary embolism was significantly reduced, and the total number of obstructive pulmonary segments, including incomplete and complete obstructive pulmonary segments, was decreased compared with that before treatment. 
Table 1

Comparison of pulmonary embolism before and after treatment

\begin{tabular}{|c|c|c|c|c|}
\hline \multirow[t]{2}{*}{ Group } & \multirow{2}{*}{$\begin{array}{l}\text { Patients } \\
\text { (n) }\end{array}$} & \multirow{2}{*}{$\begin{array}{l}\text { Obstructive } \\
\text { pulmonary segments (n) }\end{array}$} & \multicolumn{2}{|c|}{ Degree of obstruction (n) } \\
\hline & & & Incompletely & Completely \\
\hline Before treatment & 30 & 551 & 489 & 62 \\
\hline After treatment & & 301 & 260 & 41 \\
\hline
\end{tabular}

CTPA right ventricular function parameters and clinical indicators before and after treatment

The comparison of AAd, MPAd, MPAd/AAd, RPAd, LPAd, RVd/LVd, PAOI, D-dimer, cTnl, NT-proBNP, CK, LDH, AST before and after treatment in 30 patients is shown in Table 2. After thrombolytic or anticoagulant therapy, most of the patients' condition improved significantly,the comparison of CTPA before and after treatment is shown in (Fig. 1). Two patients' condition did not improve significantly after treatment, and three patients' pulmonary artery did not show obvious abnormality when they were reexamined CTPA after treatment. Compared with before treatment, some right ventricular function parameters and clinical indexes were significantly different. MPAd, RPAd, LPAd, RVd / LVd, PAOI, D-dimer, NT-proBNP, cTnl, CK, LDH, AST had significant changes after treatment $(P<0.05)$, while other parameters had no significant differences before and after treatment $(P>0.05)$. 
Table 2

Comparison of clinical data before and after treatment

\begin{tabular}{|c|c|c|c|c|}
\hline & Before & After & $\mathrm{TQZ}$ & $\mathbf{P}$ \\
\hline Age $(\overline{\mathrm{x}} \pm \mathrm{s})$ & $52.6 \pm 16.69$ & & & \\
\hline \multicolumn{5}{|l|}{ Gender [ (\%)] } \\
\hline Male & $18(60.0)$ & & & \\
\hline Female & $12(40.0)$ & & & \\
\hline AAd & $32.87 \pm 4.10$ & $32.73 \pm 4.76$ & 0.37 & 0.71 \\
\hline MPAd & $31.07 \pm 4.34$ & $28.00(26.75,31.75)$ & -2.78 & 0.04 \\
\hline MPAd/AAd & $0.95 \pm 0.12$ & $0.91 \pm 0.14$ & 2.64 & 0.13 \\
\hline RPAd & $22.60 \pm 3.39$ & $21.83 \pm 3.30$ & -2.17 & 0.03 \\
\hline LPAd & $21.33 \pm 3.04$ & $20.20 \pm 3.32$ & 3.65 & 0.02 \\
\hline $\mathrm{RVd} / \mathrm{LVd}$ & $1.20 \pm 0.45$ & $0.95 \pm 0.26$ & -3.01 & 0.01 \\
\hline PAOI & $0.50(0.33,0.50)$ & $0.35(0.07,0.48)$ & -3.14 & 0.02 \\
\hline D-dimer & $3339.50(1804.00,6684.25)$ & $830.00(443.75,1966.50)$ & -4.62 & $<0.01$ \\
\hline cTnl & $0.11(0.02,0.75)$ & $0.02(0.02,0.91)$ & -3.40 & 0.01 \\
\hline NT-proBNP & $856(294,3939)$ & $283(139,1700)$ & -2.56 & 0.011 \\
\hline CK & $70(43,173)$ & $43.50(31.00,77.25)$ & -3.00 & $<0.01$ \\
\hline LDH & $283.50(236.75,337.25)$ & $227(182.75,276.75)$ & -2.20 & 0.028 \\
\hline AST & $38.00(23.50,71.25)$ & $26.00(18.75,36.00)$ & -2.04 & 0.041 \\
\hline \multicolumn{5}{|c|}{$\begin{array}{l}\text { Abbreviations: AAd, ascending aorta diameter; MPAd, main pulmonary artery diameter; RPAd, right } \\
\text { pulmonary artery trunk diameter; LPAd,left pulmonary artery trunk diameter; RVd/LVd, the ratio of the } \\
\text { maximum short axis diameter of the right ventricle to the maximum short axis diameter of the left } \\
\text { ventricle (RVd/LVd); PAOI, pulmonary artery obstruction index; cTnl, cardiac troponin I; NT-proBNP, N- } \\
\text { terminal B-type natriuretic peptide precursor; CK, creatine kinase; LDH, lactate dehydrogenase; AST, } \\
\text { aspartate aminotransferase }\end{array}$} \\
\hline
\end{tabular}

\section{Correlation of PAOI with right ventricular function parameters and clinical indexes}

The correlation between PAOI and right ventricular function parameters and clinical indexes in PE patients is shown in Table 3. PAOI was positively correlated with RVd/ LVd, MPAd and D-dimer, and the correlation coefficient ranged from 0.281 to 0.423 . Among them, PAOI had the closest correlation with Ddimer, and the correlation coefficient was 0.423 . The correlation scatter diagram was shown in (Fig. 2); There was no significant correlation with other indexes observed. 
Table 3

Correlation of CT PAOI with imaging parameters and arterial blood gas analysis indexes

\begin{tabular}{|lll|}
\hline & PAOI & \\
\cline { 2 - 3 } & $\mathbf{r}$ & $\mathbf{P}$ \\
\hline $\mathrm{RVd} / \mathrm{LVd}$ & 0.304 & 0.018 \\
\hline AAd & 0.224 & 0.086 \\
\hline MPAd & 0.281 & 0.029 \\
\hline D-dimer & 0.423 & $<0.01$ \\
\hline cTnl & 0.239 & 0.085 \\
\hline NT-proBNP & 0.120 & 0.407 \\
\hline CK & 0.030 & 0.839 \\
\hline LDH & 0.241 & 0.099 \\
\hline AST & -0.178 & 0.199 \\
\hline $\begin{array}{l}\text { Abbreviations: PAOI, pulmonary artery obstruction index; AAd, ascending aorta diameter; MPAd, main } \\
\text { pulmonary artery diameter; RVd/LVd, the ratio of the maximum short axis diameter of the right } \\
\text { ventricle to the maximum short axis diameter of the left ventricle (RVd/LVd); cTnl, cardiac troponin }\end{array}$ \\
$\begin{array}{l}\text { l;NT-proBNP, N-terminal B-type natriuretic peptide precursor; CK, creatine kinase; LDH, lactate } \\
\text { dehydrogenase; AST, aspartate aminotransferase }\end{array}$ \\
\hline
\end{tabular}

\section{Discussion}

In recent years, the incidence of pulmonary embolism has been increasing, and the early signs and clinical symptoms are atypical. The clinical manifestations can range from occasional asymptomatic subsegmental pulmonary embolism to massive pulmonary embolism that causes cardiogenic shock and multiple organ failure [6]. The embolus obstruction of the pulmonary artery can cause patients to develop pulmonary hypertension and increase the pressure load of the right ventricle, and eventually lead to right ventricular dysfunction (RVD) in some patients, which becomes the main lethal complication of the disease. About $45 \%$ of patients with acute PE will suffer from acute right ventricular failure, and as many as $3.8 \%$ of patients will develop chronic thromboembolic pulmonary hypertension (CTEPH), and then develop severe chronic heart failure [7]. CTEPH is a relatively common and serious long-term complication in PE patients diagnosed for the first time [8, 9]. Therefore, early diagnosis, timely thrombolysis, anticoagulation therapy, and evaluation of the recovery of pulmonary vascular patency are the focus of treatment of the disease. CTPA is often used as the gold standard for the diagnosis of pulmonary embolism in clinical practice, which can realize qualitative and quantitative diagnosis while visually discovering emboli.

PAOI has important clinical value in evaluating the severity of PE patients. Qanadli et al. proposed that on the basis of CTPA diagnosis of embolism, the degree of embolism can be further quantified. The Qanadli 
index tends to be defined according to the location of the embolus and the degree of obstruction, and can better evaluate the effect before and after treatment. This study shows that after treatment in 30 patients, the embolized pulmonary segment was significantly reduced, nearly half of the incompletely blocked pulmonary artery emboli disappeared, and part of the completely blocked blood vessel returned to patency, which in turn led to a decrease in PAOI, and a significant decrease in the diameters of MPAd, RPAd, and LPAd, indicating that the patient Pulmonary hypertension has been significantly improved. Clinically, the diagnosis of RVD mainly depends on echocardiography, cardiac injury markers and the ratio of left and right ventricular diameter of CTPA, and the detection rate of the latter two methods is relatively high [10]. Because of its high diagnostic specificity, CTPA can not only accurately locate the embolus, judge the degree of embolism, but also measure the diameter of left and right cardiac cavities and arteries. Therefore, CTPA is often used to diagnose PE and obtain the signs of RVD. Previous studies have confirmed that when RVd/LVd > 1, it can be used as a standard to judge PE with right ventricular dysfunction [11,12]. Heyer et al. [13] further proposed that RVd/LVd was positively correlated with mortality to a certain extent. Therefore, judging the improvement of RVd / LVd after treatment has important guiding significance for the prognosis of patients. In this study, RVD/LVD improved the most after treatment. And research shows that PAOI and RVd/LVd are positively correlated. When PE occurs, pulmonary artery pressure increases, and then right ventricular dilation occurs. At the same time, pulmonary venous return decreases significantly, resulting in left ventricular insufficiency. Studies have proposed [14] that there is a close negative correlation between PAOI and atrium parameters, and the short diameter of RV is positively correlated with PAOI. Therefore, dilatation of the right ventricle causes the ventricular septum to be flat or even convex to the left ventricle, leading to RVd /LVd increases. This theoretically explains the reason why PAOI is closely related to the RVd/LVd value. This indicates that the larger the range of obstruction, the more severe the obstruction, and the more likely it is to cause right heart dysfunction.

D-dimer is one of cross-linked fibrin degradation products. When intravascular thrombosis is formed, fibrin is dissolved to form D-dimer, which is used clinically to reflect the important laboratory index of thrombosis and thrombolytic activity. D-dimer can be detected in the blood about 2 hours after thrombosis, and its half-life is about 8 hours. However, D-dimer is not a specific marker of coagulation activity: under all processes and conditions that produce and destroy fibrin again, the value of D-dimer may increase [15]. When D-dimer is normal, PE can be excluded. When D-dimer is raised, it is necessary to diagnose with CTPA [16]. Study has suggested [17] that there is a correlation between the level of D-dimer and the degree of pulmonary artery obstruction. And the increase of D-dimer is related to the increase of short-term and 3-month mortality. In this study, D-dimer changed significantly after treatment $(P<0.01)$, and the correlation with PAOI was significant. After the patient was treated, the level of D-dimer decreased, the number of emboli decreased or disappeared, and the PAOI value decreased. Study has shown [18] that the level of D-dimer is closely related to the incidence of all types of VTE in COVID-19 patients. Therefore, it is best to perform D-dimer assessment daily to assess the disease progression of severely infected patients. Once the D-dimer level is> $1000 \mathrm{ng} / \mathrm{mL}$, anticoagulation therapy should be 
started. Van et al. have confirmed that the threshold of D-dimer should be adjusted for patients aged 50

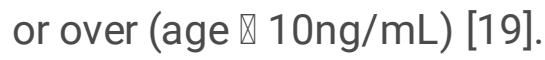

Brain natriuretic peptide is the secretion of ventricular myocardium after right ventricular dysfunction caused by right ventricular overload and dilation. CTnl is the most sensitive and specific marker of cardiomyocyte damage. Studies have shown $[20,21]$ that the cTnl level of RVD patients is significantly higher than that of patients with normal right heart function, and the increase in cTnl level is a prognostic sign of increased short-term mortality in patients with acute pulmonary embolism. Harrish et al. [22] proposed cTnl has certain value in predicting the 30-day mortality of PE patients. The combination of cTnl and quantitative CT parameters can improve the prediction of adverse clinical outcomes [23]. NTproBNP is related to the diagnosis of right ventricular dysfunction in PE patients and is an important predictor of all-cause hospitalization or short-term mortality in these patients [24]. NT-proBNP is mainly synthesized and secreted by ventricular myocytes. Changes in ventricular load and ventricular wall tension are the main conditions for increased synthesis and secretion of NT-proBNP. This provides a biological basis for the increase of NT-proBNP during acute pulmonary embolism and RVD. This study found that D-dimer, cTnl, NT-proBNP, LDH, CK had statistically significant differences before and after treatment $(P<0.05)$, while the difference in AST was relatively insignificant. Pulmonary embolism will further lead to right heart insufficiency, leading to an increase in indicators of myocardial injury. Except for D-dimer, there is no obvious correlation between other indicators and PAOI.

\section{Conclusion}

Pulmonary embolism has a high incidence rate and mortality. RVD is the main cause of death. CTPA is often used as the gold standard for clinical diagnosis of pulmonary embolism because of its high specificity, good spatial resolution and multiplanar reconstruction capability[25]. It can not only intuitively show embolic obstruction, but also make preliminary assessment of cardiac function. It is of great significance to evaluate the therapeutic effect and prognosis.

\section{Abbreviations}

PE, pulmonary embolism

CTPA, CT pulmonary angiography

PAOI, pulmonary artery obstruction index

AAd, ascending aorta diameter

MPAd, main pulmonary artery diameter

MPAd/AAd, ratio of main pulmonary artery diameter to ascending aorta diameter

RPAd, right pulmonary artery trunk diameter 
LPAd, left pulmonary artery trunk diameter

$\mathrm{RVd} / \mathrm{LVd}$, the ratio of the maximum short axis diameter of the right ventricle to the maximum short axis diameter of the left ventricle

NT-proBNP, N-terminal B-type natriuretic peptide precursor

cTnl, cardiac troponin I

AST, aspartate aminotransferase

LDH, lactate dehydrogenase

CK, creatine kinase

VTE, venous thromboembolic disease

RVD, right ventricular dysfunction

CTEPH, chronic thromboembolic pulmonary hypertension

\section{Declarations}

\section{Availability of data and materials}

The datasets generated and analysed during the current study are not publicly available due to privacy concern and patient confidentiality but are available from the corresponding author on reasonable request.

\section{Ethics approval and consent to participate}

This study was approved by The Second Affiliated Hospital of Harbin Medical University Ethics Committee. Written informed consent was obtained from all patients. We confirm that all methods were performed in accordance with the relevant guidelines and regulations. Approval number:KY-2021-100

\section{Consent for publication}

Not applicable.

\section{Competing interests}

The authors declare that they have no competing interests.

\section{Funding}


This work was supported by the National Natural Science Foundation of China. Award Number: 81701772.

\section{Authors contributions}

Meng Yuan, Xin-Yuan Cui participated in the trail design, collected and analyzed the data, and wrote the article. Chun-Xue Yang, Xin-Yang Zhang participated in the experiment design.

Jin-Ling Zhang, De-Li Zhao are the corresponding author who participated in the trial design and reviewed the article.

\section{References}

1. Cho, S.U., et al., Assessing the severity of pulmonary embolism among patients in the emergency department: Utility of RV/LV diameter ratio. PLoS One, 2020. 15(11): p. e0242340.

2. Beckman, M.G., et al., Venous thromboembolism: a public health concern. Am J Prev Med, 2010. 38(4 Suppl): p. S495-501.

3. Null, N., et al., Heart Disease and Stroke Statistics-2010 Update. Circulation, 2010. 121(7): p. e46e215.

4. Konstantinides, S. and G. Meyer, Management of acute pulmonary embolism 2019: what is new in the updated European guidelines? Intern Emerg Med, 2020. 15(6): p. 957-966.

5. Qanadli, S.D., et al., New CT index to quantify arterial obstruction in pulmonary embolism: comparison with angiographic index and echocardiography. AJR Am J Roentgenol, 2001. 176(6): p. 1415-20.

6. Meinel, F.G., et al., Predictive Value of Computed Tomography in Acute Pulmonary Embolism: Systematic Review and Meta-analysis. Am J Med, 2015. 128(7): p. 747-59.e2.

7. Bryce, Y.C., et al., Pathophysiology of right ventricular failure in acute pulmonary embolism and chronic thromboembolic pulmonary hypertension: a pictorial essay for the interventional radiologist. Insights Imaging, 2019. 10(1): p. 18.

8. Klok, F.A., et al., Diagnosis of chronic thromboembolic pulmonary hypertension after acute pulmonary embolism. Eur Respir J, 2020. 55(6).

9. Yu, Y., et al., Incidence and risk factors of chronic thromboembolic pulmonary hypertension in patients with diagnosis of pulmonary embolism for the first time in real world. Clin Respir J, 2018. 12(11): p. 2551-2558.

10. Weekes, A.J., et al., Prognostic Value of Right Ventricular Dysfunction Markers for Serious Adverse Events in Acute Normotensive Pulmonary Embolism. J Emerg Med, 2017. 52(2): p. 137-150.

11. Das, M. and J.E. Wildberger, [Diagnostics of acute pulmonary embolism : An update]. Radiologe, 2016. 56(6): p. 543-56. 
12. Furlan, A., et al., Short-term mortality in acute pulmonary embolism: clot burden and signs of right heart dysfunction at CT pulmonary angiography. Radiology, 2012. 265(1): p. 283-93.

13. Heyer, C.M., et al., Multidetector-CT angiography in pulmonary embolism-can image parameters predict clinical outcome? Eur Radiol, 2011. 21(9): p. 1928-37.

14. Faghihi, L.T., et al., The Association between the Pulmonary Arterial Obstruction Index and Atrial Size in Patients with Acute Pulmonary Embolism. Radiol Res Pract, 2019. 2019: p. 6025931.

15. Kupp, S. and J. Pöss, [Importance of biomarkers in pulmonary embolism]. Internist (Berl), 2019. 60(6): p. 571-577.

16. Kruger, P.C., et al., Pulmonary embolism: update on diagnosis and management. Med J Aust, 2019. 211(2): p. 82-87.

17. Becattini, C., et al., D-dimer for risk stratification in patients with acute pulmonary embolism. J Thromb Thrombolysis, 2012. 33(1): p. 48-57.

18. Eljilany, I. and A.N. Elzouki, D-Dimer, Fibrinogen, and IL-6 in COVID-19 Patients with Suspected Venous Thromboembolism: A Narrative Review. Vasc Health Risk Manag, 2020. 16: p. 455-462.

19. Righini, M., et al., Age-adjusted D-dimer cutoff levels to rule out pulmonary embolism: the ADJUST-PE study. JAMA, 2014. 311(11): p. 1117-24.

20. Meyer, M., et al., Benefit of combining quantitative cardiac CT parameters with troponin I for predicting right ventricular dysfunction and adverse clinical events in patients with acute pulmonary embolism. Eur J Radiol, 2012. 81(11): p. 3294-9.

21. Becattini, C., M.C. Vedovati and G. Agnelli, Prognostic value of troponins in acute pulmonary embolism: a meta-analysis. Circulation, 2007. 116(4): p. 427-33.

22. Nithianandan, $H_{\text {., }}$ et al., Applying rigorous eligibility criteria to studies evaluating prognostic utility of serum biomarkers in pulmonary embolism: A systematic review and meta-analysis. Thromb Res, 2020. 195: p. 195-208.

23. Apfaltrer, P., et al., Prediction of adverse clinical outcome in patients with acute pulmonary embolism: evaluation of high-sensitivity troponin I and quantitative CT parameters. Eur J Radiol, 2013. 82(3): p. 563-7.

24. Cavallazzi, R., et al., Natriuretic peptides in acute pulmonary embolism: a systematic review. Intensive Care Med, 2008. 34(12): p. 2147-56.

25. Palm, V., et al., Acute Pulmonary Embolism: Imaging Techniques, Findings, Endovascular Treatment and Differential Diagnoses. Rofo, 2020. 192(1): p. 38-49.

\section{Figures}




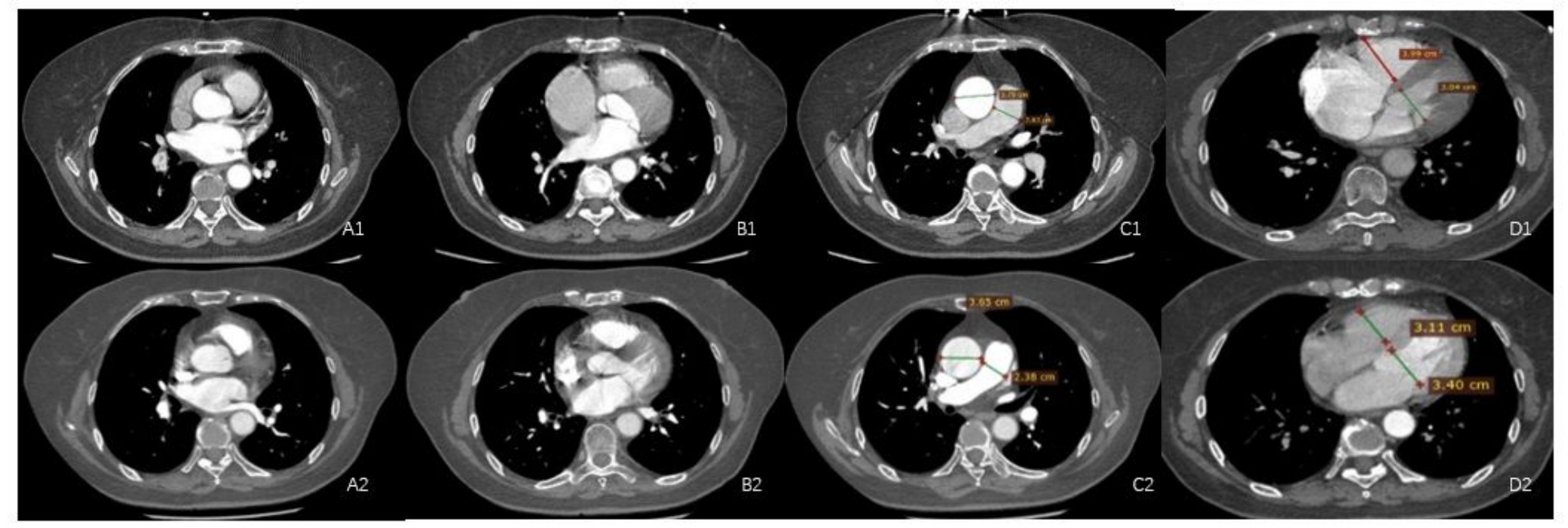

\section{Figure 1}

a1, a2: CTPA images before and after treatment showed that the right inferior pulmonary artery embolism was filled with contrast agent and the lumen was unobstructed; c1, c2: CTPA images before and after treatment showed that the left inferior pulmonary artery embolism was filled with contrast agent and the lumen was unobstructed; c1, c2: MAPd, AAd before and after treatment, the diameter and pressure of large vessels decreased after treatment; $\mathrm{c} 1, \mathrm{c} 2$ : RV/LV were 1.43 and 0.93 before and after treatment, respectively. The ratio after treatment was $<1$, and the right ventricular load was reduced.

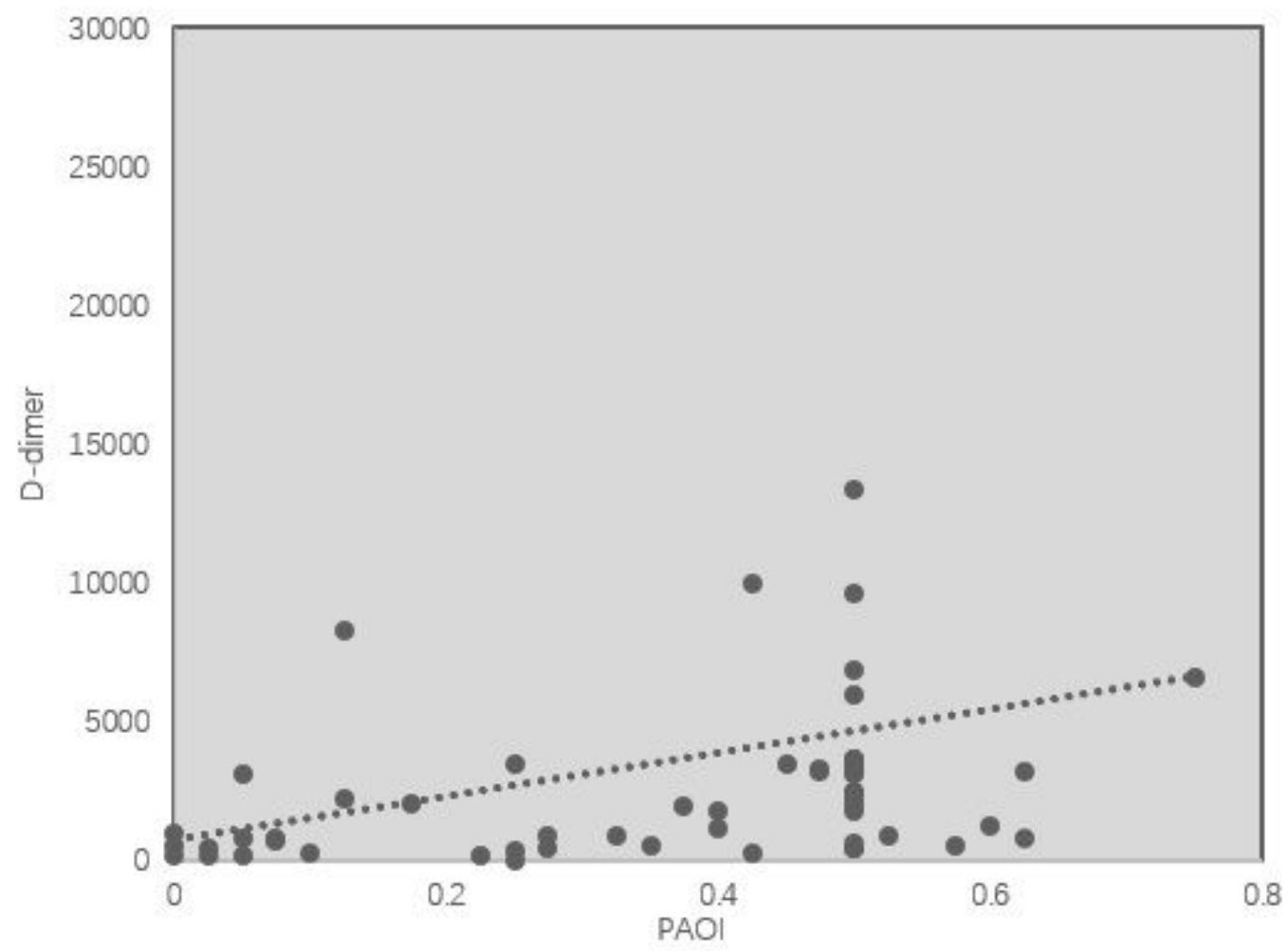

Figure 2 
scatter plot of correlation between Paio and D-dimer

Page $14 / 14$ 